\title{
Producción científica de las universidades peruanas en temas de salud durante los años 2014 y 2015
}

Victor Hugo Moquillaza Alcántara 1,a , Mercedes Joselyn Nuñez Ochoa 2,b

\section{RESUMEN}

La producción científica universitaria a nivel latinoamericano aún se encuentra muy por debajo del nivel internacional; sin embargo, no existen artículos publicados que describan esta situación en el Perú. Por ello, mediante un análisis de la base secundaria del Consejo Nacional de Ciencia, Tecnología e Innovación Tecnológica (Concytec), se buscó determinar la producción científica en temas de salud en revistas indizadas y no indizadas durante los años 2014 y 2015. Se encontró que la mediana oscila entre 1 y 2 artículos publicados por universidad al año, con un un incremento de la producción entre el 10 al 98,16\% en las revistas indizadas y las no indizadas nacionales, mientras que la producción en revistas no indizadas internacionales tuvo un decremento del 42,86 \%.

Palabras clave: Investigación; Investigación biomédica; Perú; Universidades (Fuente: DeCS BIREME).

\section{Scientific production of Peruvian universities on health topics during 2014 and 2015}

\section{ABSTRACT}

The university scientific production at the Latin-American level is still far below the international level. In spite of this, there are no publications describing this situation in Peru. Therefore, it was sought to determine the scientific production on health topics in indexed and non-indexed journals during the years 2014 and 2015 through an analysis of the secondary database of the National Council for Science, Technology and Technological Innovation (CONCYTEC). It was found that the median ranged between 1 and 2 articles published by university per year, with the production increasing between 10 and $98,16 \%$ in national indexed and non-indexed journals, and decreasing in $42,86 \%$ in international non-indexed journals.

Keywords: Research; Biomedical research; Peru; Universities (Source: MeSH NLM).

1. Universidad Peruana Cayetano Heredia. Lima, Perú.

2. Universidad Nacional Mayor de San Marcos. Lima, Perú.

a. Estudiante de Maestría en Informática Biomédica.

b. Interna de Obstetricia. 


\section{INTRODUCCIÓN}

La visibilidad científica es entendida como la cantidad y calidad de publicaciones de una institución con el objetivo de trascender en la comunidad científica, $\mathrm{y}$ es relevante para las universidades debido a que proporciona indirectamente un indicador de su calidad educativa (1). Sin embargo, existe una marcada diferencia entre la producción científica de los países denominados "en desarrollo" y aquellos "desarrollados"; caracterizada en los primeros por la ausencia de inversión, insuficiente asignación de recursos estatales y escasa visibilidad de la producción universitaria ${ }^{(2)}$.

Scimago, red de análisis bibliométrico de Scopus, reporta que desde 1996 los países que mayor producción presentan en temas médicos son Estados Unidos, Reino Unido, Alemania y Japón. Los países de habla española con mayores documentos publicados son España (358 000), y, muy por debajo, México (58 000) y Argentina $(44000)^{(3)}$.

En Argentina se realizó un análisis bibliométrico sobre la producción científica de 14 universidades en Córdova, se encontró un predominio de publicaciones internacionales en detrimento de las revistas y congresos nacionales ${ }^{(4)}$.

A diferencia de ello, Maz-Machado en Colombia publicó también un análisis bibliométrico, donde encontró que la producción se centraba en el nivel local, con un $81,91 \%$ de manuscritos publicados en revistas nacionales. En 12 años, Colombia produjo 15 302 artículos, de los cuales 3873 correspondieron a la Universidad Nacional de Colombia (UNC); con una la media nacional de 1275 artículos/año ${ }^{(5)}$. La UNC produjo 322,75 artículos/año ${ }^{(6)}$.

Castro et al. reportan que en la Facultad de Odontología de la Universidad Nacional Mayor de San Marcos del Perú, el promedio de producción por alumno es de $0,04 \pm 0,2$ artículos y que el $61,1 \%$ de los estudiantes perciben una falta de asesoramiento adecuado en metodología y redacción científica ${ }^{(7)}$.

Taype señala que los investigadores de la Universidad de San Martín de Porres han publicado 57 artículos en revistas indizadas en el campo de las ciencias médicas, alcanzando 16 publicaciones durante el año $2013^{(8)}$.

La elaboración de estrategias y políticas para prevenir y manejar los problemas de salud se basa en la evidencia existente; sin embargo, esta evidencia se produce mayormente en países ajenos a Latinoamérica. Esto amerita la promoción y el fortalecimiento de la producción de investigación y evidencias que midan y analicen la problemática en el nivel local y nacional y abordar los retos necesarios para que se incremente la producción científica en el país ${ }^{(2)}$.

\section{EL ESTUDIO}

Se realizó un análisis de la base secundaria de Concytec denominada I Censo Nacional de Investigación y Desarrollo a Centros de Investigación 2016, la cual es de libre acceso en la página web de esta institución. La base presenta el gasto e inversión en investigación, el personal dedicado a investigación y los resultados de la investigación (producción científica) de las instituciones del Perú.

Se filtraron los datos pertenecientes a instituciones de educación superior privadas y estatales $(\mathrm{N}=451)$ que desarrollaron producción científica en temas de ciencias médicas y de la salud, obteniendo 68 universidades durante el 2014 y 83 durante el 2015.

Se tomaron en consideración los criterios de publicación en revistas científicas indizadas y no indizadas, nacionales o internacionales. Para la presentación de datos se realizó previamente la evaluación de la normalidad de las variables a estudiar mediante el uso de la prueba de ShapiroWilk.

\section{RESULTADOS}

Se contabilizaron un total de 1029 artículos publicados en temas de ciencias médicas y de la salud durante el lapso de los 2 años. Todas las variables consideradas en el estudio presentaron una distribución no normal: número de publicaciones en revistas indizadas y número de publicaciones en revistas no indizadas, a nivel nacional e internacional.

La tabla 1 muestra la publicación en revistas indizadas nacionales por parte de las universidades peruanas mostrando un incremento del $10 \%$ entre el año 2014 y el 2015, con 140 y 154 artículos publicados respectivamente. La mediana de publicaciones por universidad fue de 2 artículos por año. 
Tabla 1. Número de publicaciones científicas en temas médicos y de la salud de las universidades peruanas

\begin{tabular}{|c|c|c|c|c|c|c|c|c|c|}
\hline & \multicolumn{4}{|c|}{2014} & \multicolumn{4}{|c|}{2015} & \multirow[t]{2}{*}{2015} \\
\hline & $\mathrm{Me}$ & $\mathrm{P}_{25}$ & $\mathrm{P}_{75}$ & Total & $\mathrm{Me}$ & $\mathrm{P}_{25}$ & $P_{75}$ & Total & \\
\hline \multicolumn{10}{|c|}{ Revista indizada } \\
\hline Nacional & 2 & 1 & 6 & 140 & 2 & 0 & 5 & 154 & $+10 \%$ \\
\hline Internacional & 1 & 0 & 4 & 163 & 1 & 0 & 7 & 323 & $+98,16 \%$ \\
\hline \multicolumn{10}{|c|}{ Revista no indizada } \\
\hline Nacional & 1 & 1 & 4 & 98 & 1 & 1 & 10 & 129 & $+31,63 \%$ \\
\hline Internacional & 0 & 0 & 0 & 14 & 0 & 0 & 0 & 8 & $-42,86 \%$ \\
\hline
\end{tabular}

En contraposición, a nivel internacional se presentaron 163 artículos (Me: 1, RIQ: 4) durante el 2014 y 323 (Me: 1, RIQ: 7) durante el 2015, lo cual representó un incremento del 98,16\% (Figura 1).

Al evaluar el número de publicaciones en revistas no indizadas nacionales se encontró que se publicaron 98 artículos (Me:1, RIQ: 3) y durante el 2015 fueron 129 (Me:1, RIQ: 9), con lo cual se mostró un incremento del $31,63 \%$ en la producción científica dentro de esta categoría (Tabla 1, figura 2).



Figura 1. Producción científica de las universidades peruanas en revistas indizadas durante los años 2014 y 2015

Así mismo se determinó que dentro de las publicaciones en medios no indizados internacionales durante el 2014 se presentaron 14 artículos y durante el año 2015 tan solo 8 publicaciones, presentando así un decremento del $42,86 \%$ en la producción científica no indizada internacional (Figura 2). 


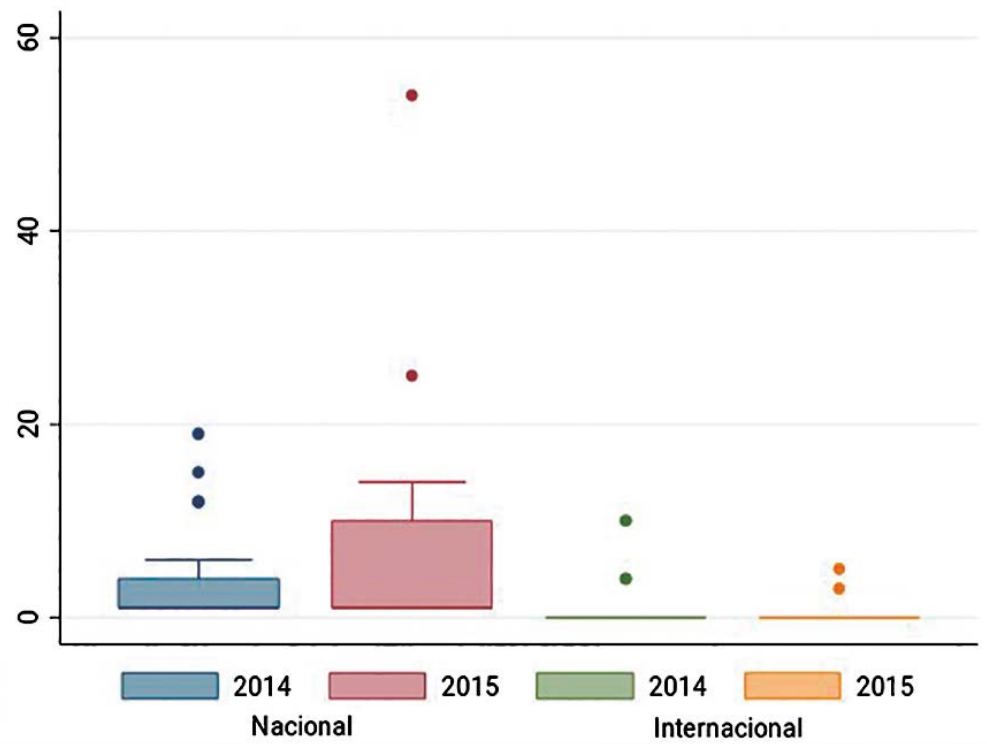

Figura 2. Producción científica de las universidades peruanas en revistas no indizadas durante los años 2014 y 2015

Finalmente, en la figura 3 se muestra la evaluación del número de publicaciones de acuerdo al número de universidades. Se halló una disminución de universidades que publicaron un artículo durante el año $2014(\mathrm{n}=6)$ frente al año $2015(\mathrm{n}=5)$. El número de universidades que publicaron 2 artículos por año se mantuvieron durante los años 2014 y 2015 (n=9). Destacó la presencia de una universidad ubicada en Lima, sin identificación de su nombre en la base de datos de Concytec, que registra 500 publicaciones durante los años consecutivos evaluados.

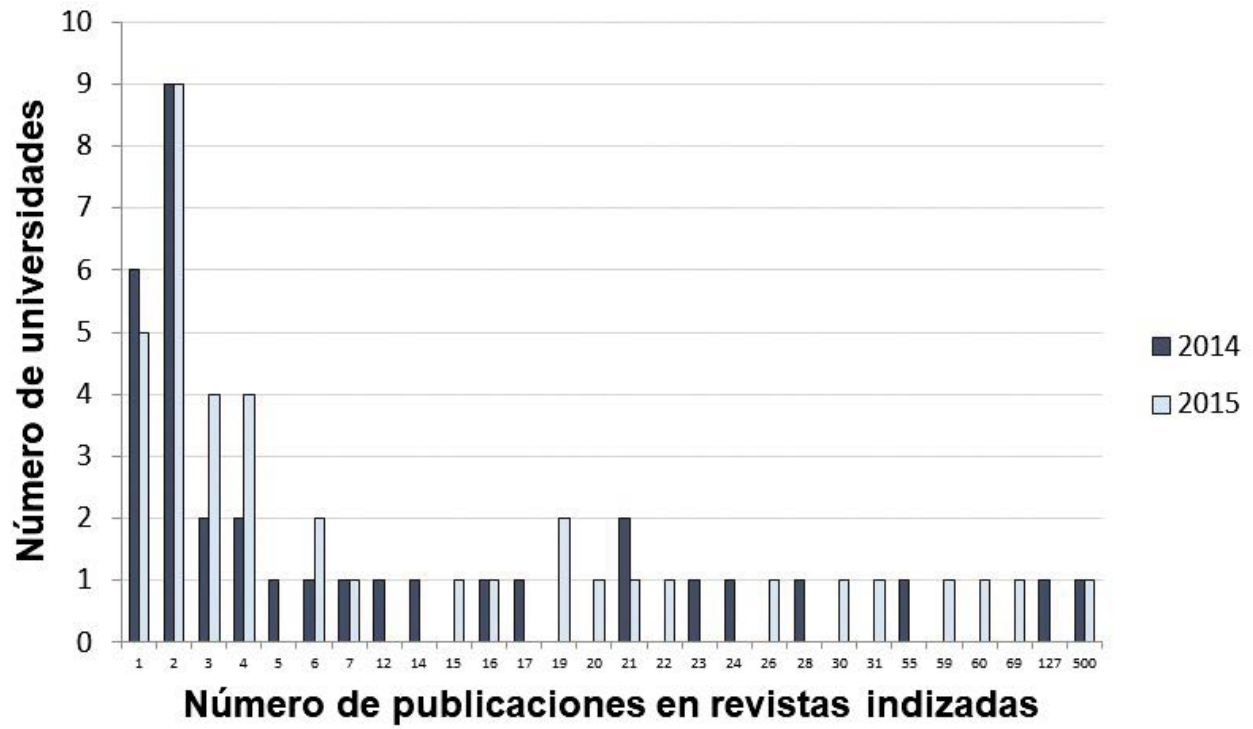

Figura 3. Número de publicaciones en revistas indizadas por universidades durante los años 2014 y 2015 


\section{DISCUSIÓN}

La producción nacional por año en temas médicos y de la salud rodea los 514 artículos por año, lo cual dista con lo presentado en diversos países. España, que lidera la producción en países de habla castellana produce, por año, aproximadamente, 17 mil artículos ${ }^{(3)}$, así también duplica la producción del Perú con aproximadamente 1275 artículos por año ${ }^{(5)}$.

Se encuentra la misma realidad al analizar la producción por universidad, la cual, según la base de datos de Concytec, es en promedio de 2 artículos por año por universidad, situación preocupante si la comparamos con la Universidad Nacional de Colombia, por ejemplo, la cual produce más de 300 artículos al año ${ }^{(6)}$. Estos promedios son bajos debido a que muchas universidades en el Perú aún no incluyen la investigación dentro de sus actividades. Sin embargo, existen otras universidades, como por ejemplo la Universidad de San Martín de Porres, que han logrado generar más de 10 artículos por año ${ }^{(8)}$.

Bustos, en Argentina, describe que en este país existe un mayor predominio por la publicación en revistas indizadas internacionales ${ }^{(4)}$. Es destacable el incremento de la producción entre los años 2014 y 2015, que alcanza un crecimiento de 98,16 \% en alguna categoría; sin embargo, aún existe una producción muy pobre si se aspira destacar científicamente a nivel internacional.

Es importante considerar que, como en toda base secundaria, pueden existir sesgos en el llenado correcto de los datos o falsificación de información en lo reportado por algunas universidades, lo cual puede generar error. Aun así, cabe destacar el trabajo de Concytec al brindar herramientas para el análisis e interpretación de los datos, por lo cual los autores de este estudio recomiendan seguir trabajando en esta área con la finalidad de mostrar evidencias de la realidad peruana en cuanto a ciencia, tecnología e innovación.

\section{REFERENCIAS BIBLIOGRÁFICAS}

1. Turpo JE, Medina GE. Producción intelectual y visibilidad científica. Apuntes Universitarios. 2013; 2(2):9-18.

2. Vera AA, Escobar FH, Montealegre M, González FM. Relaciones entre indicadores, política científica internacional y producción científica en América Latina y el Caribe: Una mirada retrospectiva a los procesos de internacionalización en la Universidad Surcolombiana. Entornos. 2007; 1(20):7-15.

3. SJR - International Science Ranking: 1996 - 2016. Disponible en: https: //www.scimagojr.com/

4. Bustos E, Centeno A, Rapela MV. Bibliometric analysis of the scientific production of researchers with projects approved by the Science and Technology Office of the Universidad Nacional de Córdoba (Argentina): 1996-1999. Transinformação. 2003; 15(2):231-43.

5. Maz-Machado A, Jiménez-Fanjul NN, Villaraga ME. La producción científica colombiana en SciELO: un análisis bibliométrico. Rev Interam Bibliot. 2016; 39(2): 111-9.

6. Escobar-Córdoba F, Eslava-Schmalbach J, Gómez-Duarte OG. Producción científica de los departamentos de la Facultad de Medicina de la Universidad Nacional de Colombia entre los años 2000 y 2012. Rev Fac Med. 2016; 64(2): 189-98.

7. Castro Y, Sihuay-Torres K, Perez-Jiménez V. Producción científica y percepción de la investigación por estudiantes de odontología. Educ Med. 2018; 19 (1): 19-22.

8. Taype-Rondán Á, Luque L. Producción científica en Scopus de la Universidad de San Martín de Porres, Lima, Perú. Horiz Med. $2014 ; 14(4): 37-42$.

Fuentes de financiamiento:

Este artículo ha sido financiado por los autores.

Conflictos de interés:

Los autores declaran no tener ningún conflicto de interés.

\section{Correspondencia:}

Victor Hugo Moquillaza Alcántara

Dirección: Condominio Los Nogales, Ed.1 Dto. 304, El Agustino. Lima, Perú.

Teléfono: 982065404

Correo electrónico: victor.moquillaza@upch.pe

$$
\begin{aligned}
& \text { Recibido: } 17 \text { de mayo de } 2018 . \\
& \text { Evaluado: } 29 \text { de mayo de } 2018 . \\
& \text { Aprobado: } 05 \text { de junio de } 2018 .
\end{aligned}
$$

๑ La revista. Publicado por Universidad de San Martín de Porres, Perú.

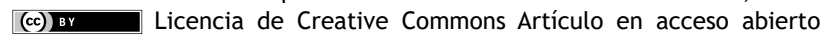
bajo términos de Licencia Creative Commons Atribución 4.0 Internacional. (http://creativecommons.org/licenses/by/4.0/)

\section{ORCID iDs}

Victor Hugo Moquillaza Alcántara. Mercedes Joselyn Nuñez Ochoa

https: / /orcid.org/0000-0002-0362-907X https://orcid.org/0000-0003-1284-2830 